\title{
The artificial hip fails
}

\section{Samuels A*}

Formerly Reader in law in the University of Southampton, formerly President of the Medico-legal Society, UK

The orthopaedic surgeon advised and recommended the patient to have a prosthetic artificial hip, chose the make of the artificial hip, and duly fitted it. The operation appeared to be a success. But after three years, due to metal fatigue, the artificial hip fractured and cast debris, and had to be removed and replaced. The patient could sue the surgeon and the hospital for clinical negligence in the ordinary way. He could sue the seller for selling goods not of satisfactory quality and not fit for the purpose. He could sue the manufacturer for negligence in putting defective goods on the market. There is liability for defective goods under the Consumer Protection Act 1987 part I product liability s 3, principally concerned with safety, the law deriving from the EU Directive $85 / 375$. A product is defective if the safety of the product is not such as persons generally are entitled to expect. Safety is a relative concept. The claimant will have to show that the hip was defective, he suffered injury, and the injury was caused by the defect. All relevant circumstances must be taken into account, to include how the product has been marketed, the get-up, the use of any product mark, instructions for use and any warnings Wilkes v Deputy International Ltd [2016] EWHC 3096 (QB), [2018] 2 WLR 531, (2017) BMLR 91.

The liability of the manufacturer and the seller is not absolute or strict. The safety of the product is required to be what persons generally are entitled to expect. The expectation of the individual or particular patient cannot be the test; he knows nothing of the technology he just expects the hip to work. The orthopaedic surgeon is an expert in orthopaedics, he understands anatomy, he has fitted many artificial hips, and he knows which ones work well. But he may not be an expert on the design or the metals or other materials used in the manufacturer, nor is he any sort of mechanical engineer. However, many orthopaedic surgeons have made it their business to understand the qualities of the metals and other materials, the strengths and weaknesses, the engineering, the design, the statistics; and indeed, some have been closely involved in design and some types of artificial hips carry the name of a surgeon or a medical department. So, there may be a degree of shared knowledge and responsibility.

The issue is not whether or not the manufacturer is at fault. The issue is whether the product objectively measured up to the legal standard as set by the judge. That depends upon the relevant circumstances in the particular case. Experts will be needed on materials and mechanics, and on the medical aspects of the effects of metal or other material entering the internal circulatory system. The words defect and defective are not statutorily defined, so the judge has to carry out a balancing act in the particular case. This technique makes predictability difficult.

Did the manufacturer comply with the Medical Devices Regulations 2002 SI 618 and the regulatory requirements?

Did the manufacturer comply with the industry and product standards, such as those set by the British Standards Institute BSI?

Did the manufacturer meet the product specifications?
What has been the failure rate, e.g. $1 \%$ or $5 \%$ or whatever? Experience of usage must be of real significance. A very low failure rate might indicate that anticipating or predicting or guarding against a defect might be extremely difficult. An artificial hip is not expected to last for ever, but failure before 10 years or even 15 years is very rare. Bad luck cannot be prevented. Everyone concerned should ensure that they are well informed regarding the known failure rate.

What was the risk, and every product must carry some risk, set against the benefit? An artificial hip must be very beneficial to the patient needing one. The principal responsibility for the safety and wellbeing of the patient rests with the surgeon. But complete protection against failure simply is not attainable. Age is inevitably a factor.

What was the cost? In one sense the consumer gets what he pays for, but whatever the cost the consumer of a medical product, particularly an artificial hip, must be entitled at the very least to an appropriate minimum quality standard.

Did the manufacturer give a proper warning of the risks? Every patient is different, the human variable, and there are some well-known risks. For example, trouble can arise from excessive weight, excessive physical exercise, certain types of manual labour, and uneven individual gait, especially from trouble with the other hip. Post-operation behaviour on the part of the patient must be the principal responsibility of the surgeon, and the patient should follow instructions and advice.

Many thousands of patients have been fitted with the pinnacle system, which has an inherent propensity to shed particular debris, which normally causes no problem, but in a small percentage of cases causes an adverse immunological reaction. Some three hundred sufferers needed revision surgery after a few years. They brought a collective or class or group action against the manufacturer. Was the product "defective"? Colin Gee v Depuy International Ltd [2018] EWHC 1208 (QB).

The basic or standard test had to be applied. Was the standard of safety at the level normally to be expected or tolerated by the normal patient or client or customer? Did the product, the artificial hip, carry any risks beyond the normal risks? Particularisation was impossible to prevent. Particularisation normally did not lead to adverse immunological reaction; although it was known that this could happen. The manufacturer could not be expected to produce a product that would last for ever, would never cause a problem. Normal particularisation and normal wear and tear were inevitable. In 10 to 15

${ }^{\star}$ Correspondence to: Samuels A, Formerly Reader in law in the University of Southampton, formerly President of the Medico-legal Society, UK, E-mail: alec. samuels@btinternet.com

Received: June 01, 2018; Accepted: June 11, 2018; Published: June 14, 2018 
years some revision surgery was to be expected, in normal and sound artificial hips. The hips in question, the model in question, was no better and no worse than any other comparable hips on the market. There was no abnormal risk of damage. The legitimate expectations of the patients were fulfilled. The product was not defective.

\section{Medical liability}

What about the liability of the surgeon and the hospital? The surgeon may be liable for negligence in choosing and fitting the artificial hip, but this seems very unlikely if it was normal and suitable. But he may be liable if he did not warn the patient of any risks that the patient either asked about or would reasonably be expected to want to know about, and the risk of replacement at some time in the future would seem to fall in that category Montgomery v Lancashire Health Board [2015] UKSC 11.
The patient will not have acquired the new hip directly, it will have been acquired by the hospital; but there will be a professional intermediary, the orthopaedic surgeon and his team, who should be appropriately experienced and skilled, able to identify a visible or obvious defect, and carry out all the appropriate procedures, such as post-operative $\mathrm{x}$ rays and monitoring.

\section{Consumer Rights Act 2015}

Under a contract to supply goods those goods must be of satisfactory quality, in a state and condition fit for all the purposes for which goods of that kind are usually supplied, safe and durable Consumer Rights Act 2015 s 9, fit for any particular purpose expressly or by implication made known to the trader s 10, match the description s 11 , and giving all information required by regulation to be given to the consumer s 12 .

Copyright: $\odot 2018$ Samuels A. This is an open-access article distributed under the terms of the Creative Commons Attribution License, which permits unrestricted use, distribution, and reproduction in any medium, provided the original author and source are credited. 